\title{
Insights from an autism imaging biomarker challenge: promises and threats to biomarker discovery
}

Authors: Nicolas Traut ${ }^{1,9} \dagger$, Katja Heuer ${ }^{1,4,9} \uparrow$, Guillaume Lemaître ${ }^{2,3} \uparrow$, Anita Beggiato ${ }^{1,11}$, David Germanaud $^{5}$, Monique Elmaleh ${ }^{6}$, Alban Bethegnies ${ }^{7}$, Laurent Bonnasse-Gahot ${ }^{12}$, Weidong Cai ${ }^{10}$, Stanislas Chambon ${ }^{16}$, Freddy Cliquet ${ }^{1}$, Ayoub Ghriss ${ }^{8}$, Nicolas Guigui ${ }^{5}$, Amicie de Pierrefeu ${ }^{5}$, Meng Wang ${ }^{14,15}$, Valentina Zantedeschi ${ }^{13}$, Alexandre Boucaud ${ }^{2,3}$, Joris van den Bossche ${ }^{2,3}$, Balázs Kegl $^{17}$, Richard Delorme ${ }^{1,11}$, Thomas Bourgeron ${ }^{1}$, Roberto Toro ${ }^{1}+$, Gaël Varoquaux ${ }^{2,18}+*$

$\dagger$ : equal contribution

+ : equal contribution

\section{Affiliations:}

1. Institut Pasteur, Université de Paris, Département de neuroscience, F-75015 Paris, France.

2. Parietal, Inria, Saclay, France.

3. Paris-Saclay Center for Data Science, Université Paris Saclay, Saclay, France.

4. Max Planck Institute for Human Cognitive and Brain Sciences, Leipzig, Germany.

5. Neurospin CEA, Saclay, France.

6. Department of Radiology, Robert Debré, APHP, Paris, France.

7. Hosa.io, Paris, France.

8. University of Colorado, Boulder, US.

9. Center for Research and Interdisciplinarity (CRI), Université Paris Descartes, Paris, France.

10. Stanford University School of Medicine, Palo Alto, US.

11. Child and Adolescent Psychiatry Department, Robert Debré, APHP, Paris, France.

12. Centre d'Analyse et de Mathématique Sociales, EHESS, CNRS, PSL, Paris, France.

13. Univ Lyon, UJM-Saint-Etienne, CNRS, Institut d'Optique Graduate School, Laboratoire Hubert Curien UMR 5516, F-42023, Saint-Etienne, France.

14. Brainnetome Center and National Laboratory of Pattern Recognition, Institute of Automation, Chinese Academy of Sciences, Beijing 100190, China.

15. University of Chinese Academy of Sciences, Beijing 100049, China.

16. Rythm.co, 75009 Paris.

17. Huawei, Paris.

18. Montréal Neurological Institute, McGill, Montréal, Canada.

*To whom correspondence should be addressed: gael.varoquaux@inria.fr 


\begin{abstract}
MRI has been extensively used to identify anatomical and functional differences in Autism Spectrum Disorder (ASD). Yet, many of these findings have proven difficult to replicate because studies rely on small cohorts and are built on many complex, undisclosed, analytic choices. We conducted an international challenge to predict ASD diagnosis from MRI data, where we provided preprocessed anatomical and functional MRI data from $>2,000$ individuals. Evaluation of the predictions was rigorously blinded. 146 challengers submitted prediction algorithms, which were evaluated at the end of the challenge using unseen data and an additional acquisition site. On the best algorithms, we studied the importance of MRI modalities, brain regions, and sample size. We found evidence that MRI could predict ASD diagnosis: the 10 best algorithms reliably predicted diagnosis with AUC $\sim 0.80$ - far superior to what can be currently obtained using genotyping data in cohorts 20-times larger. We observed that functional MRI was more important for prediction than anatomical MRI, and that increasing sample size steadily increased prediction accuracy, providing an efficient strategy to improve biomarkers. We also observed that despite a strong incentive to generalise to unseen data, model development on a given dataset faces the risk of overfitting: performing well in cross-validation on the data at hand, but not generalising. Finally, we were able to predict ASD diagnosis on an external sample added after the end of the challenge (EU-AIMS), although with a lower prediction accuracy (AUC $=0.72$ ). This indicates that despite being based on a large multisite cohort, our challenge still produced biomarkers fragile in the face of dataset shifts.
\end{abstract}

\title{
Highlights
}

- MRI can provide reliable biomarkers for ASD

- Because of overfitting, models with excellent apparent performance on public data failed to generalise

- The 10 best algorithms used classic machine learning methods

- Biomarker development needs blinded evaluation.

- Prediction performance should reach $\mathrm{AUC}=0.83$ on samples with $10 \mathrm{k}$ subjects

\section{Introduction}

Autism Spectrum Disorder (ASD) is a life-long neurodevelopmental disorder which affects more than $1 \%$ of the population. Its severity differs vastly among individuals, however, they all share persistent deficits in social communication and restricted, repetitive and stereotyped behaviours. ASD is heritable, and influenced by common genetic variation as well as rare mutations (Krumm et al. 2015; Bourgeron 2015; Sandin et al. 2017; Weiner et al. 2017). Early intervention has a 
significant positive impact on the patient's outcome, which makes early diagnosis a research priority (Dawson et al. 2010).

Magnetic resonance imaging (MRI) is an important tool to explore the brain of individuals with ASD: it is a widely available, fast, and non-invasive method to measure brain anatomy and function. By providing detailed measurements of an individual's brain, MRI brings the promises of precision psychiatry, adapting therapy to patients (Insel 2014). But can MRI be used to characterise ASD in general? For more than 30 years, MRI studies have described anatomical and functional differences between individuals with ASD and unaffected controls: enlarged brain volume and cortical surface area (Piven et al. 1995; E. Courchesne et al. 2001), decreases in brain volume and neocortical thinning during adolescence and adulthood (Lange et al. 2015; Zielinski et al. 2014), smaller corpus callosum (Egaas et al. 1995; Wolff et al. 2015), abnormal cerebellar volume (Courchesne 1987; Hodge et al. 2010; Fatemi et al. 2012), and global and regional increases and decreases in functional connectivity (Just 2004; Belmonte 2004; Di Martino et al. 2014a; Cheng et al. 2017).

Many of these findings are, however, controversial and have proven difficult to replicate (Haar et al. 2016; Lefebvre et al. 2015; Traut et al. 2018; Picci et al. 2016; Mohammad-Rezazadeh et al. 2016). Most studies have relied on sample sizes far too small to reach reliable conclusions sometimes just a few dozen subjects, and up to a few hundreds at most. They lack replication and reanalysis on independent data. This is particularly problematic because of the multitude of parameters involved in each analysis which could substantially alter the results (Carp 2012; Power et al. 2012; Poldrack et al. 2017): acquisition sequence, subject motion, software packages, pre-processing workflow, etc.

Rather than focusing on the detection of specific regional differences between cases and controls, brain-imaging features can be combined into a biomarker of ASD answering the question: can diagnostic status be inferred from MRI data? Machine-learning provides important techniques to build and characterise such biomarkers. Yet, machine-learning studies of ASD are most often based on the analysis of a single sample, without validation of the findings in an independent sample. The community recognises today that establishing the validity of a biomarker needs a fully independent assessment on new data, otherwise its accuracy cannot be trusted (Woo et al. 2017; Poldrack et al. 2019) as it may arise from overfitting, circular analysis (Kriegeskorte et al. 2009) or researchers' degrees of freedom (Ioannidis 2005). This is particularly critical for machine learning approaches, where classifiers trained on data from one sample may be unable to generalise to additional samples (Ecker et al. 2015). Publication incentives lead researchers to seek and report the best prediction accuracy. For brain-imaging biomarkers of ASD, publications have reported accuracies above 95\% (Bi et al. 2018). If that were true, the accuracy of those algorithms would be equivalent to the inter-rater reliability of clinical assessment by human 
experts (kappa $=95 \%)$ which defines the gold-standard for discrimination of ASD versus other development disorders (Klin et al. 2000). But how trustworthy is the evaluation of biomarkers such as those in (Bi et al. 2018), given that the whole study - biomarker extraction and validation - was done on only 50 ASD patients and 42 non-ASD controls? Peer-review is not sufficient to assess the analytic choices as even minor variants lead to large differences in observed prediction accuracy, though these are unlikely to reveal true improvements (Varoquaux 2018).

To ground solid conclusions on ASD neuroimaging, several international consortia have been constituted such as ABIDE (Autism Brain Imaging Data Exchange, Di Martino et al. 2014b; Di Martino et al. 2017), EU-AIMS (European Autism Interventions - A Multicentre Study for Developing New Medications, Murphy and Spooren 2012) or the IBIS Network (Infant Brain Imaging Study, Hazlett et al. 2012), increasing sample sizes through data sharing. They extend the amount and quality of the data collected through harmonisation efforts. Recent analysis across cohorts has shown that ASD is significantly associated with changes in functional connectivity (Holiga et al. 2019). But are these changes large enough to ground reliable prediction to new sites, despite heterogeneity in imaging techniques and populations recruited?

The study we present built upon these large cohorts, and framed the extraction of biomarkers as an open, international challenge to predict ASD from the largest MRI dataset currently available - more than 2,000 individuals. A data-science prediction challenge of this type can provide conclusive evidence on the ability of MRI to detect ASD, because it is based on a blind evaluation of the results in addition to relying on a large sample. Furthermore, it isolates the development of the analysis pipeline from its evaluation. Challengers did not have access to validation data, which allowed us to test the ability of the algorithms to generalise to unseen data, including data acquired in different centers.

\section{Materials and methods}

\section{Brain imaging dataset}

The brain imaging dataset combined data from the public Autism Brain Imaging Data Exchange (ABIDE) I and II datasets (Di Martino et al. 2014a; Di Martino et al. 2017) and a private dataset from the Robert Debré Hospital (RDB) in Paris, France. ABIDE provides open access to functional and anatomical MRI data for 2,156 subjects. The RDB dataset contained data from 247 subjects, 56 of whom were also part of the ABIDE II project: we excluded these duplicate subjects from the private dataset.

We extracted anatomical features from the anatomical MRI: regional brain volumes, cortical thickness, and surface area; and extracted time-series signals from the resting-state functional 
MRI. To derive these individual measurements, the data were processed with standard neuroimaging tools: Freesurfer (Fischl et al. 1999), FSL (Woolrich et al. 2009), and AFNI (Cox 1996). We split the total dataset into public and private datasets, aiming at balancing the age and sex distributions (Fig. 1 provides demographic information for the public and private datasets). A total of 2,117 subjects were included: 947 ASD and 1,170 controls. We did not exclude subjects based on quality control, but provided challengers with quality control scores obtained from visual inspection by 3 experts. The public dataset contained data from 1,150 subjects: 549 ASD and 601 controls. The private dataset contained data from 967 subjects: 398 ASD and 569 controls. The public dataset was a subset of ABIDE I and II, while the private dataset combined data from ABIDE I, II and RDB. The dataset should capture well the clinical and methodological heterogeneity of ASD neuroimaging. The ABIDE dataset was collected by 24 different centres worldwide, and spans an age range from 5 to 64 years old (median 13.8 years old). All subjects had intellectual quotients within the normal range ( $97 \%$ have full IQ $>80), 80 \%$ of patients and $90 \%$ of controls were right-handed. The cohort was composed of $80 \%$ males. This diversity should allow classification algorithms to generalise, preventing them from specialising in a particular type of data or age range. Further demographic information for the ABIDE I and II datasets is provided in their corresponding publications (Di Martino et al. 2014a; Di Martino et al. 2017).

\section{Study design: a data-science prediction challenge}

Description of the challenge: We launched the challenge inviting data scientists to submit algorithms to predict ASD diagnostic from provided MRI data. The challenge lasted 3 months and attracted 146 challengers. We awarded money prizes to the 10 best challengers. These were determined after the closure of the competition, by assessing how well the algorithms would predict ASD diagnostic in a private, unseen dataset.

The ABIDE data can be openly distributed, which enabled us to provide a rich dataset on which challengers could tune their algorithms. To facilitate access to the data, we provided challengers with a "starting kit" giving a proof-of-concept predictive model on the data (extracted on standard brain atlases). Challengers were then able to develop their own prediction algorithms, which they submitted using a Web interface. The code was executed on our central server. The challengers never had access to the private dataset. They used machine-learning techniques tuned on the public dataset and submitted the corresponding code to the central server which evaluated them in the private dataset. 
a. Subjects distribution

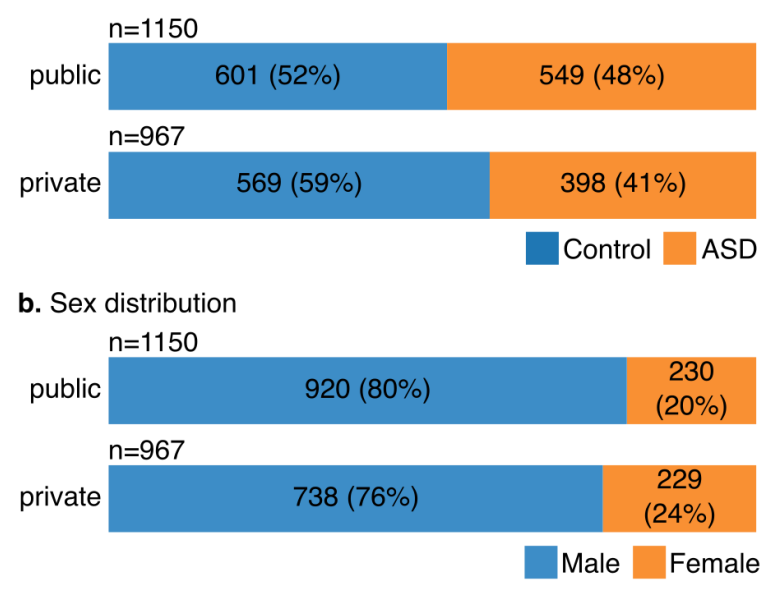

c. Age distribution

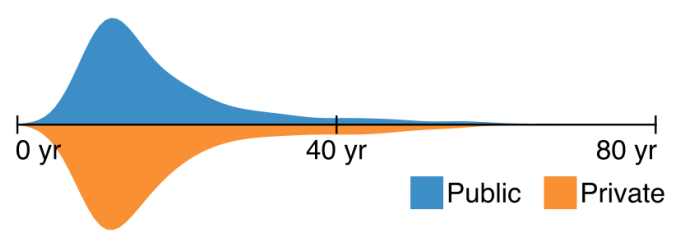

d. Site distribution for ABIDE I

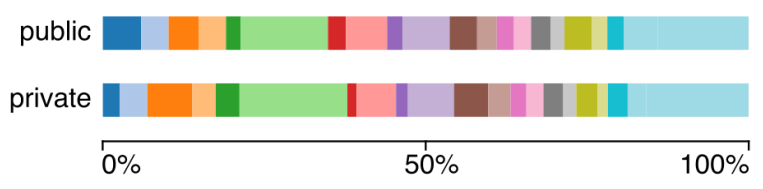

e. Site distribution for ABIDE II

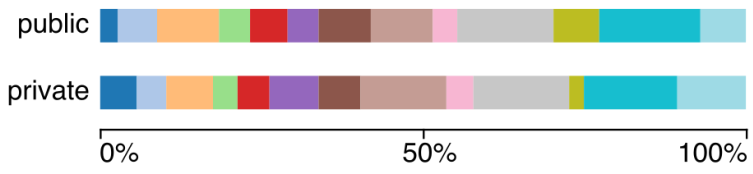

Figure 1. Subjects, sex, age, and site distributions. The distributions of the number of cases and controls, their sex ratio, age and scanning site were similar in the public and private datasets.

To measure the quality of the predictions, we used a standard metric: the area under the receiver operating characteristic curve (ROC-AUC). This measure summarises various detection tradeoffs, for example, favouring few false negatives to the cost of false positives in the case of a screening study, or the converse, in the case of a confirmatory study. Prediction at chance level gives an AUC of 0.5, while perfect prediction gives an AUC of 1.

Statististical analysis strategy: After the closure of the challenge, we analysed the 10 best submissions to understand the factors driving their predictions. Considering that these machine-learning algorithms captured the best possible predictive biomarkers given our brain-imaging cohort, we varied the data on which they were applied. Each time, we fit the algorithms anew on the data, to extract the corresponding biomarkers, and measured the prediction accuracy on the private dataset. First, we applied them to different imaging modalities: only functional MRI, or only anatomical MRI. Second, we varied the number of available subjects, to measure the importance of the sample size. Finally, we investigated the importance 
of different brain regions by removing those that appeared as most discriminative and attempting to extract biomarkers from the rest of the data. To compute which regions were the most discriminative, we used the absolute value of the model's coefficients when the algorithm used a linear model, and the feature importance when the algorithm used a random forest. We obtained a region-level summary of functional-connectivity biomarkers by associating to every region the sum of the importance of its connections, a measure of node strength. Region-level importance was then turned into a brain map characterising the spatial distribution of the discriminant information.

\section{MRI preprocessing and signal extraction}

Anatomical MRI: Anatomical MRI was preprocessed and segmented using FreeSurfer v6.0. We extracted three kinds of anatomical features: (i) mean regional cortical thickness, (ii) cortical surface area of regions parcellated with the Desikan-Killiany Atlas (Desikan et al. 2006), and (iii) volumes of subcortical regions segmented with the FreeSurfer atlas (Fischl et al. 2002).

Resting-state functional MRI: Resting-state functional MRI captures brain activity and functional connectivity. It is typically studied via a functional-connectome: a matrix capturing interactions between brain regions. We provided time series extracted on a variety of atlases after standard fMRI preprocessing using the pipeline from the FC1000 project (which includes slice-time interpolation, motion correction, coregistration to anatomic data, normalisation to template space). The brain parcellations and atlases used were: (i) BASC parcellations with 64, 122, and 197 regions (Bellec et al. 2010); (ii) Ncuts parcellations (Craddock et al. 2012); (iii)

Harvard-Oxford anatomical parcellations; (iv) MSDL functional atlas (Varoquaux et al. 2011); and (v) Power atlas (Power et al. 2011).

\section{Challenge organisation}

Organisation of the challenge: We launched the challenge on May $1^{\text {st }} 2018$ and closed it on July $1^{\text {st }} 2018$. The challenge attracted about 146 participants accounting for a total of about 720 submissions. We awarded money prizes to the 10 best challengers. Challengers were ranked based on the ROC-AUC score of their submission computed on the private dataset. We framed the challenge problem by providing: (i) a public dataset, (ii) a standard way to assess the submission, and (iii) a starting kit. For this purpose, we used the RAMP (Rapid Analytics and Model Prototyping) workbench. Participants submitted their solutions on the RAMP website (https://ramp.studio). During the challenge we provided to the participants the cross-validated score computed on the public dataset. At the end of the challenge, we asked each participant to select a single submission. This submission was trained (fitted) on the public dataset and 
evaluated on the private dataset hidden from the participants. Participants were ranked based on the score of these submissions computed on the private dataset.

Challenge platform: RAMP is an online data science tool used to organise challenges. RAMP enables us to easily compare and reproduce predictive experiments. It can be used on the user's computer or online: the former is for developing predictive models while the latter assesses their predictive accuracy. A RAMP "starting kit" is a placeholder where we define the data-science problem: we provide the datasets, the metric, and the model validation technique. Participants can focus on the development of their machine-learning predictive model. We also provide examples to help participants understand the challenge. The RAMP website was used to evaluate the solutions of the participants (i.e., predictive models): participants submit their code and the website trains (fits) and evaluates them. We deployed, trained, and tested the full workflow on Amazon Web Services. Note that participants can also train and test their models locally. However, they only have access to the public dataset to test their models. We rely on Python and its rich ecosystem. Participants were free to use any Python library to build their machine learning model.

Data and code availability: The public data, the code of the ten best submissions, the MRI preprocessing scripts and the scripts used to generate the figures are available at https://github.com/neuroanatomy/autism-challenge.

\section{Results}

\section{Participation to the challenge}

We received 589 submissions from 61 teams. To assess external validity of the biomarkers (Steyerberg and Harrell 2016), the private dataset contained $>200$ subjects not included in ABIDE from the Robert Debré Hospital in Paris, France (RDB). We selected the 10 submissions which performed the best (taking only one submission per team) as the winning ones, and gave money prizes to the submitters. After close inspection we discovered that we forgot to remove 56 subjects of the RDB dataset which were also in the ABIDE dataset. For the post-hoc analyses presented here, we removed those duplicated subjects from the private dataset to avoid artificially inflating the prediction score. The ranking between the 10 best submissions remained relatively stable after the removal of the duplicate subjects (Spearman correlation: $r s=0.7697, p$ $(2$-tailed $)=0.00922)$. 
The combination of the 10 best models provided a good predictor of ASD diagnosis.

We combined the 10 best models using a blending approach (Caruana et al. 2004) to produce a probability for ASD diagnosis for each individual. These probabilities were in general higher for patients than for controls (Fig. 2a). The receiver operating curve (ROC, Fig. 2b) represents the quality of these predictions for different tradeoffs of sensitivity and specificity, which can be summarised by the area under the curve (AUC). The combined predictor reached an AUC of 0.80, which is considered as a good discrimination level (Hosmer and Lemeshow 2000). Used as a screening test, the predictor would correctly detect $88 \%$ of the individuals with ASD at the cost of misclassifying $50 \%$ of controls as patients. Used as a confirmatory diagnostic test, the predictor would detect $25 \%$ of the individuals with ASD and only $3 \%$ of controls would be misclassified as patients. Predictions were also good (even slightly better) on the RDB subjects which were absent from the $\mathrm{ABIDE}$ dataset (median $\mathrm{AUC}=0.809$ versus $\mathrm{AUC}=0.768$ - Fig. $2 \mathrm{~d}$ ).

After the challenge, we used an additional dataset from the EU-AIMS project to evaluate the ability of the blended predictor to generalise. Performance on this additional data was slightly worse than on our external site $(\mathrm{AUC}=0.721)$, revealing that the predictor was still fragile to distribution shifts despite having been extracted from multiple sites.

Further increases in sample size should lead to increased prediction accuracy

The analysis of the learning curve showed that prediction accuracy was not reaching a plateau and should keep improving by increasing the number of subjects (Fig. 2e). Extrapolating on this increase, we estimate that prediction accuracy could reach an $\mathrm{AUC}=0.83$ if a dataset of 10,000 subjects were available.

\section{Functional MRI had the strongest impact on prediction accuracy}

Studying separately the prediction accuracy obtained for each imaging modality showed that functional MRI contributed more to prediction than anatomical MRI (AUC $=0.79$ using only functional MRI, versus $\mathrm{AUC}=0.66$ using only anatomical MRI, Fig. 2c). Incorporating age and sex information only had a small influence on prediction accuracy (increasing AUC to 0.80).

\section{Regions distributed over the entire brain contributed to prediction}

We analysed the functional MRI biomarkers to highlight the most discriminative brain regions. For this, we ranked brain regions by their importance in the 10 best models. Overall, prediction relied on regions distributed over the entire brain, with regions around the precuneus appearing to be the most important (Fig. 3a). We assessed the relative importance of the different brain 
medRxiv preprint doi: https://doi.org/10.1101/2021.11.24.21266768; this version posted November 28, 2021. The copyright holder for this preprint (which was not certified by peer review) is the author/funder, who has granted medRxiv a license to display the preprint in perpetuity.

It is made available under a CC-BY-NC 4.0 International license .

regions by progressively removing those that contributed the most and extracting new biomarkers. Prediction accuracy remained high even after removing up to $50 \%$ of the most important brain regions (see Fig. 3b). This suggests that the biomarkers captured information distributed over the entire brain.

a. Predicted probability of ASD for patients and controls

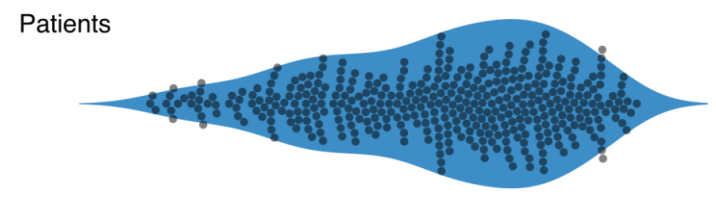

Controls

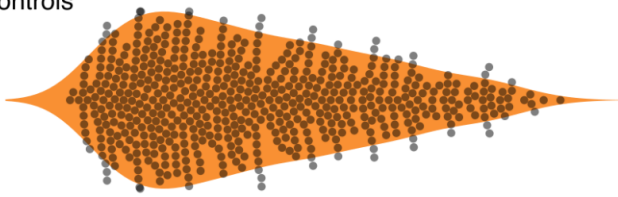

0.0

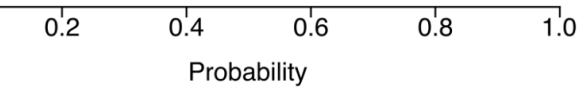

b. Prediction accuracy $(A \cup C=0.80)$

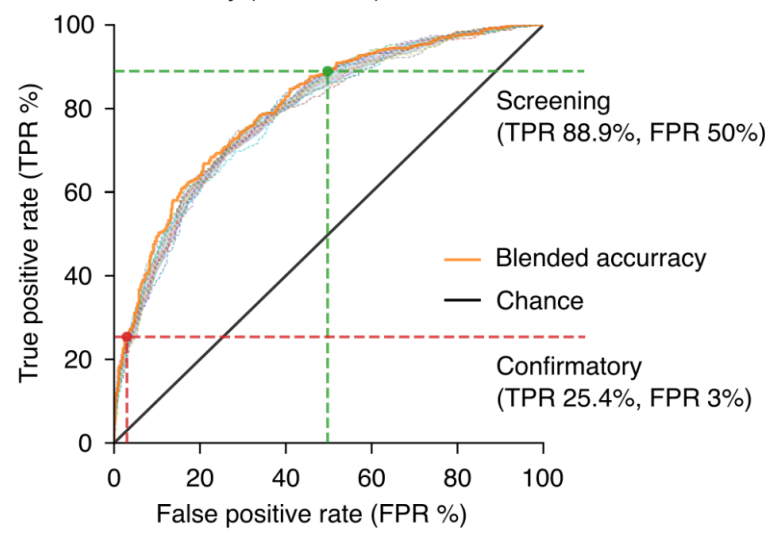

c. Importance of different data modalities

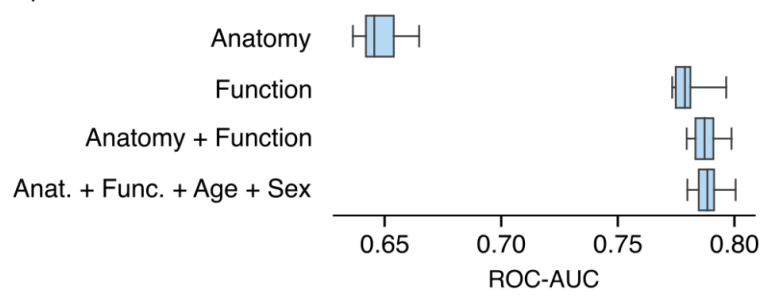

d. Heterogeneity across sites (external validity)

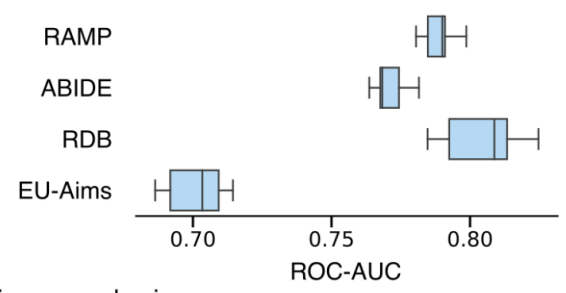

e. Prediction for various sample sizes

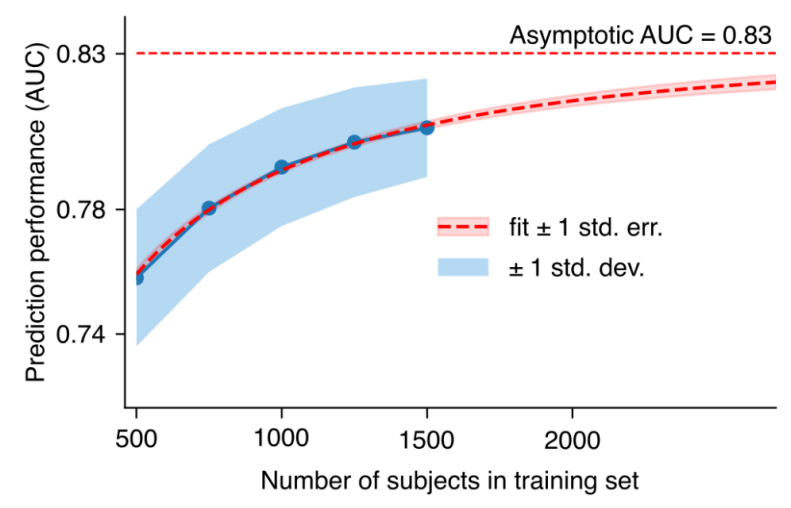

Figure 2. Performance analysis using the 10 best submissions of the challenge. (a) Predicted probability for patients and controls to be classified with ASD. (b) Prediction accuracy obtained by combining all available data modalities (anatomical and functional MRI, age, and sex). Using the biomarkers for screening purposes led to a True Positive Rate (TPR) of $88.9 \%$ for a False Positive Rate (FPR) of 50\%. Enforcing a low FPR (3\%) to make a confirmatory analysis led to a True Positive Rate of $25.4 \%$. (c) Importance of the data modalities to predict ASD. Functional MRI provided a higher discriminative power than the other available data (population indicators and anatomical MRI data). (d) Data heterogeneity across sites was not a roadblock: methods generalised to data from new sites, unseen during training. (e) Prediction accuracy by varying the number of subjects in training. Prediction accuracy did not reach a plateau with the current number of subjects available. Increasing the number of subjects improves the discriminative power of the methods. The current trend suggests an increase of prediction performance to 0.83 for a dataset of 10,000 subjects. 
a. Importance of regions for functional MRI

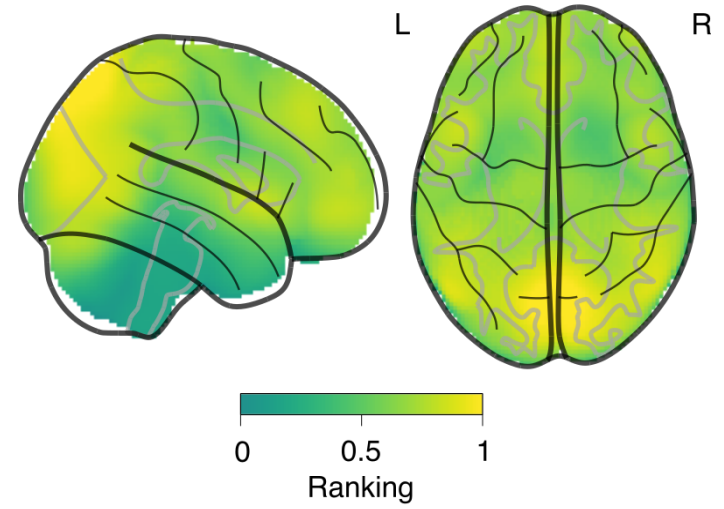

b. Change in accuracy after region removal

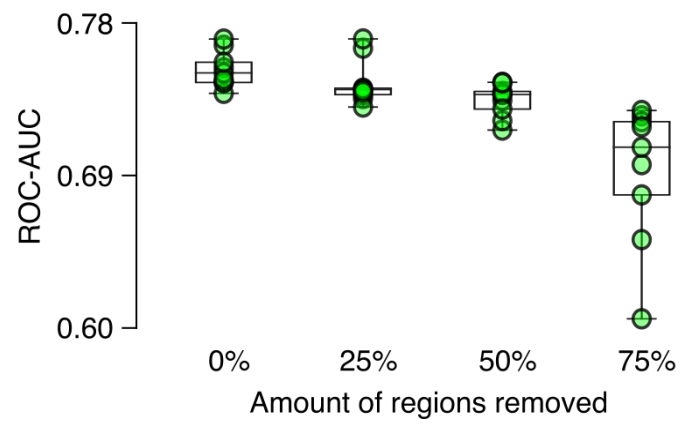

\section{Discussion and conclusions}

\section{MRI can provide reliable biomarkers for ASD}

The results of our challenge conclusively demonstrate that MRI is a powerful and reliable method to study ASD. Interestingly, it is the size of the cohort - not interindividual heterogeneity - that is the main factor limiting prediction accuracy. This can be seen from the fact that aggregating data across sites with different recruitment policies led to a steady increase in prediction accuracy. Increasing sample size is so far the best strategy for achieving further progress: better predictive power and better spatial localisation of the biomarkers. We project that a population of 10,000 individuals should allow us to reach the maximum prediction accuracy that a simple case-control design can achieve. At this stage, the adoption of a dimensional approach to model ASD should lead to further improvements in the prediction of clinical status.

The results of our challenge suggest that MRI provides an important source of information for the study of ASD, complementary to that obtained, for example, through genetic exploration. Recent reports show that polygenic risk scores (PRS), aiming to predict case-control status from genome-wide common variants (Purcell et al. 2009), can explain $2.45 \%$ of the risk variance on 
the observed scale (Nagelkerke pseudo-R ${ }^{2}$ ) in a group of 13,076 ASD cases and 22,664 controls (Grove et al. 2019). Considering a disease prevalence of $1.2 \%$, PRS should capture $1.13 \%$ of the risk variance of ASD on the liability scale (Lee et al. 2012). As a matter of comparison, the AUC of 0.80 that we obtain from MRI corresponds to an $\mathrm{R}^{2}$ of $19.1 \%$ on a liability scale with the same disease prevalence (Wray et al. 2010). We can expect that larger genetic samples for ASD should increase prediction accuracy, as the proportion of risk variance captured by common genetic variants is estimated to be $11.8 \%$ on the liability scale (Grove et al. 2019). The important gap with prediction accuracy is explained by the fact that the effects of common genetic variants are highly diluted across the genome, and therefore difficult to estimate individually. It may be informative to consider the case of schizophrenia - a neurodevelopmental disorder with overlapping genetic aetiology. In schizophrenia, PRS obtained from approximately 35,000 cases and 45,000 controls captured $\sim 15 \%$ of the risk variance on the observed scale (Nagelkerke pseudo- ${ }^{2}$ ) and $\sim 7 \%$ on the liability scale with an AUC of 0.70 (Schizophrenia Working Group of the Psychiatric Genomics Consortium 2014). Our MRI-based prediction of ASD provides a higher accuracy than what PRS can provide for schizophrenia, with a much smaller cohort. MRI-based prediction may have the additional benefit of providing longitudinal information which could be important to track disease progression. In combination, genetic and MRI information could provide a powerful tool to predict and understand ASD risk.

\section{Classic machine learning methods provided the best results}

The starting kit we presented transformed the resting state fMRI time series from the MSDL atlas to a tangent correlation matrix (Varoquaux et al. 2010) and regressed stacked values from the matrix and anatomical values with a L1-penalised logistic regression. The starting kit combined the predictions from the functional and anatomical MRI with a meta-classifier based on a logistic regression. The transformation from the functional MRI time series to a tangent correlation matrix was adopted by all submissions; however, the choice of atlas was variable. Seven submissions used time series from several atlases, while three submissions used only one atlas. In addition to the transformation to a tangent correlation matrix, one submission transformed to a standard correlation matrix and another transformed to a partial correlation matrix. One submission performed dimensionality reduction using a principal component analysis (PCA). Six submissions used logistic regression as a first layer predictor, two used linear c-support vector classification (SVC) and the two others a combination of different methods. Three submissions obtained directly a single prediction whereas the remaining seven obtained several predictions, which were combined using a logistic regression for five of them, average for one them, and a majority vote for the last one.

Challengers used a variety of methods, which included machine-learning techniques ranging from simple logistic regressions to complex graph-convolutional deep-learning models. 
medRxiv preprint doi: https://doi.org/10.1101/2021.11.24.21266768; this version posted November 28, 2021. The copyright holder for this preprint (which was not certified by peer review) is the author/funder, who has granted medRxiv a license to display the preprint in perpetuity.

It is made available under a CC-BY-NC 4.0 International license .

Inspection of the individual scores showed that deep-learning techniques displayed strong overfits: good performance on the public dataset but poor generalisation to the private dataset. On the opposite, many algorithms which used simpler approaches had a stable prediction performance when applied to new data, displaying prediction accuracies between $\mathrm{AUC}=0.7$ and 0.8 which did generalise to the private dataset. The submissions which led to reliable predictions had some methodology in common. In particular, they used linear support vector machines or logistic regression to compute the combination of the different signals - anatomical and functional - that best discriminated patients from controls. In addition, several submissions combined signals from multiple atlases using a stacking strategy (Wolpert 1992).

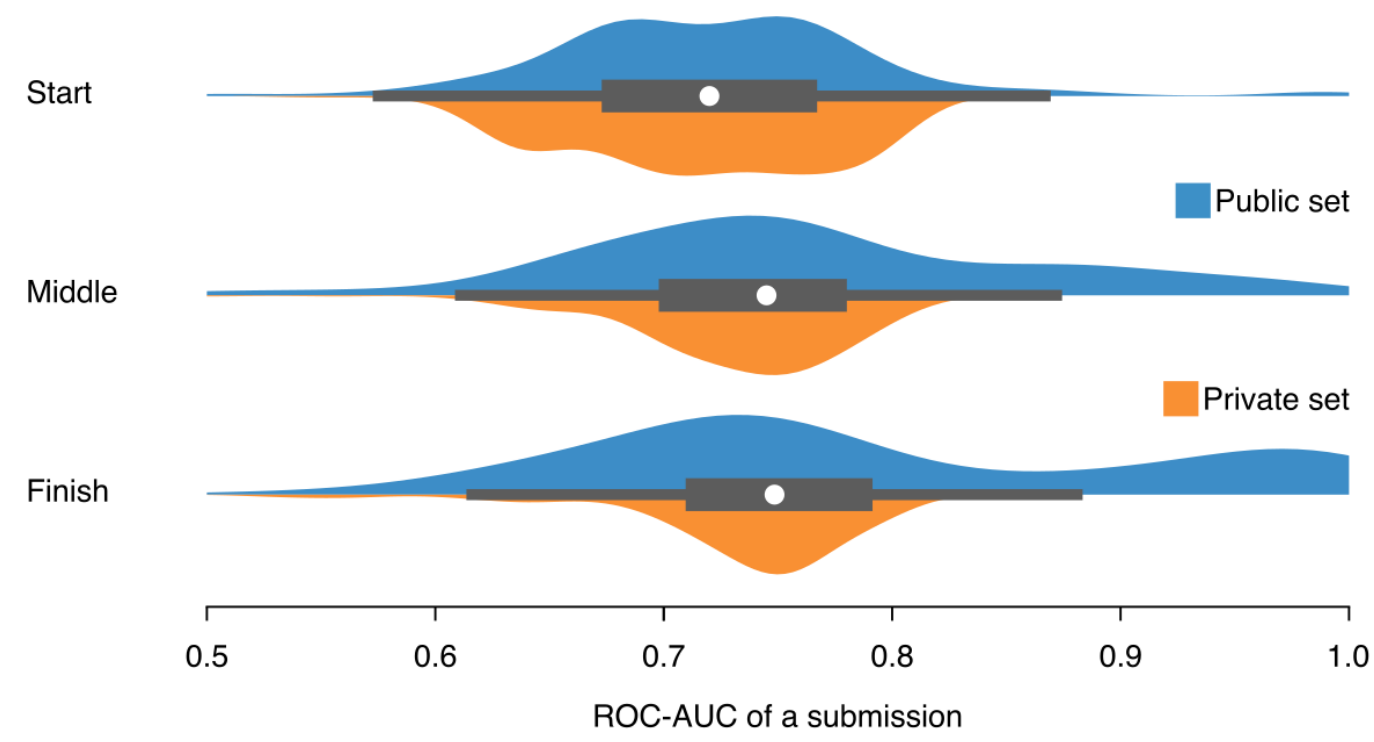

Figure 4. Score during the competition. The evolution of the scores of all submissions on the public and private datasets suggest that participants' work led mostly to increased prediction performance in the public dataset without comparable increases in the private dataset. 


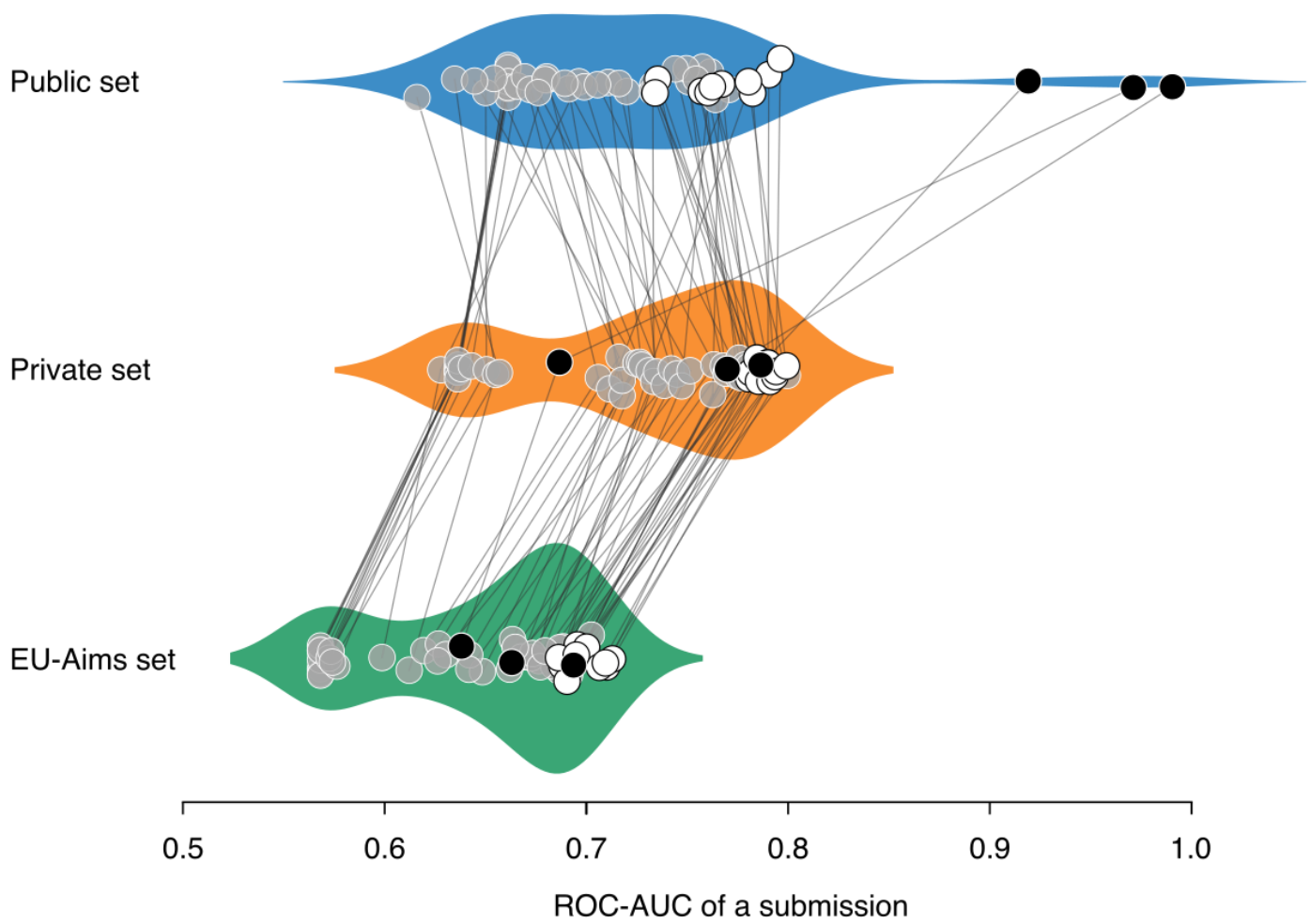

Figure 5. Excellent performance on the public dataset was misleading. The score of each final submission to the challenge is represented as a point on the public and the private dataset. The prediction on the public dataset was measured with 8 random splits (with $20 \%$ of the data left out), the standard machine-learning procedure to measure prediction performance. Extreme prediction scores in the public dataset $(\mathrm{AUC}>0.8)$ did not generalise to the private dataset. By contrast, algorithms with conservative scores $(0.6<\mathrm{AUC}<0.8)$ did predict the private dataset equally well.

Most improvement came from using atlases with larger numbers of regions.

To speed up the computation, we presented a starting kit with the smaller atlas for resting state functional MRI. We saw that switching to larger atlases considerably increased the AUC: switching from the MSDL atlas (39 regions) to the craddock_scorr_mean atlas (249 regions) increased the AUC of the functional starting kit from 0.655 to 0.778 and the AUC of combined anatomy and functional from 0.716 to 0.790 . The best submission we had obtained an AUC of 0.799 - an increase of less than 0.009 .

\section{Evaluation without a blind validation set is at risk of severe optimism}

Our challenge highlights that a trustworthy development of biomarkers must include the evaluation on a new dataset, blinded to the analysts. In our challenge, participants had no interest in developing algorithms that would give optimistic results on the public dataset, as they knew 
that they would be evaluated on the private dataset. Nevertheless, comparing the prediction quality on the public dataset to that on the private dataset (Figs. 4 and 5) clearly showed that the best predictions on the public dataset were too good to be true, and did not carry over to the unseen, private dataset. This is likely because the challengers made their analytic choices or trained hyperparameters to improve the prediction score that they measured on the public dataset (Fig. 4). As a result, they obtained seemingly-excellent predictions, but which likely relied on noise in the public data and did not generalise to the private dataset. Indeed, the techniques used in machine-learning to measure prediction performance, such as cross-validation, are not completely robust to systematic exploration of analytic choices (Varoquaux 2018): To fully trust the prediction accuracy of biomarkers, these must be validated externally, as in our challenge. However, when testing external validity on a third, completely new data (EU-AIMS, see Fig. 5), the ranking of the models stayed roughly similar as on the private dataset, a pattern already reported on kaggle challenges (Roelofs et al. 2019). The blind assessment was necessary to select methods that extract good ASD biomarkers, and to reliably quantify a prediction accuracy.

\section{Better biomarkers bring new opportunities}

Robust predictive MRI biomarkers open several opportunities for clinical and scientific research: Predictive MRI biomarkers enable longitudinal follow-ups and prospective epidemiology. Infants at risk of ASD could be scanned longitudinally, which could allow us to develop early biomarkers useful when behaviour is not a sufficient basis for diagnosis. Collecting and sharing brain-imaging data for 10,000 individuals at risk of ASD is well within the reach of a community effort. Such a large number would enable a substantial hold-out sample, and hence precise characterisation of the biomarkers. As predictive biomarkers reach an excellent prediction accuracy, the hope is that they will narrow down on the discriminant information. Hence, increases in prediction performance will also reveal more precise information on the neural correlates of ASD.

\section{References}

Bellec, Pierre, Pedro Rosa-Neto, Oliver C. Lyttelton, Habib Benali, and Alan C. Evans. 2010. "Multi-Level Bootstrap Analysis of Stable Clusters in Resting-State fMRI." NeuroImage 51 (3): 1126-39.

Belmonte, M. K. 2004. "Autism and Abnormal Development of Brain Connectivity." Journal of Neuroscience. https://doi.org/10.1523/jneurosci.3340-04.2004.

Bi, Xia-An, Yingchao Liu, Qin Jiang, Qing Shu, Qi Sun, and Jianhua Dai. 2018. "The Diagnosis of Autism Spectrum Disorder Based on the Random Neural Network Cluster." Frontiers in Human Neuroscience. https://doi.org/10.3389/fnhum.2018.00257.

Bourgeron, Thomas. 2015. "From the Genetic Architecture to Synaptic Plasticity in Autism Spectrum Disorder." Nature Reviews. Neuroscience 16 (9): 551-63.

Carp, Joshua. 2012. "The Secret Lives of Experiments: Methods Reporting in the fMRI Literature." NeuroImage. https://doi.org/10.1016/j.neuroimage.2012.07.004.

Caruana, Rich, Alexandru Niculescu-Mizil, Geoff Crew, and Alex Ksikes. 2004. "Ensemble Selection from 
Libraries of Models." In Proceedings of the Twenty-First International Conference on Machine Learning, 18. ICML '04. New York, NY, USA: Association for Computing Machinery.

Cheng, Wei, Edmund T. Rolls, Jie Zhang, Wenbo Sheng, Liang Ma, Lin Wan, Qiang Luo, and Jianfeng Feng. 2017. "Functional Connectivity Decreases in Autism in Emotion, Self, and Face Circuits Identified by Knowledge-Based Enrichment Analysis." NeuroImage 148 (March): 169-78.

Courchesne, E., C. M. Karns, H. R. Davis, R. Ziccardi, R. A. Carper, Z. D. Tigue, H. J. Chisum, et al. 2001. "Unusual Brain Growth Patterns in Early Life in Patients with Autistic Disorder: An MRI Study." Neurology 57 (2): $245-54$.

Courchesne, Eric. 1987. "Abnormal Neuroanatomy in a Nonretarded Person With Autism." Archives of Neurology. https://doi.org/10.1001/archneur.1987.00520150073028.

Cox, R. W. 1996. “AFNI: Software for Analysis and Visualization of Functional Magnetic Resonance Neuroimages." Computers and Biomedical Research, an International Journal 29 (3): 162-73.

Craddock, R. Cameron, G. Andrew James, Paul E. Holtzheimer 3rd, Xiaoping P. Hu, and Helen S. Mayberg. 2012. "A Whole Brain fMRI Atlas Generated via Spatially Constrained Spectral Clustering." Human Brain Mapping 33 (8): 1914-28.

Dawson, Geraldine, Sally Rogers, Jeffrey Munson, Milani Smith, Jamie Winter, Jessica Greenson, Amy Donaldson, and Jennifer Varley. 2010. "Randomized, Controlled Trial of an Intervention for Toddlers with Autism: The Early Start Denver Model." Pediatrics 125 (1): e17-23.

Desikan, Rahul S., Florent Ségonne, Bruce Fischl, Brian T. Quinn, Bradford C. Dickerson, Deborah Blacker, Randy L. Buckner, et al. 2006. "An Automated Labeling System for Subdividing the Human Cerebral Cortex on MRI Scans into Gyral Based Regions of Interest." NeuroImage 31 (3): 968-80.

Di Martino, Adriana, David O'Connor, Bosi Chen, Kaat Alaerts, Jeffrey S. Anderson, Michal Assaf, Joshua H. Balsters, et al. 2017. "Enhancing Studies of the Connectome in Autism Using the Autism Brain Imaging Data Exchange II." Scientific Data 4 (March): 170010.

Di Martino, A., C-G Yan, Q. Li, E. Denio, F. X. Castellanos, K. Alaerts, J. S. Anderson, et al. 2014a. "The Autism Brain Imaging Data Exchange: Towards a Large-Scale Evaluation of the Intrinsic Brain Architecture in Autism." Molecular Psychiatry 19 (6): 659-67.

—. 2014b. "The Autism Brain Imaging Data Exchange: Towards a Large-Scale Evaluation of the Intrinsic Brain Architecture in Autism." Molecular Psychiatry 19 (6): 659-67.

Ecker, Christine, Susan Y. Bookheimer, and Declan G. M. Murphy. 2015. "Neuroimaging in Autism Spectrum Disorder: Brain Structure and Function across the Lifespan." Lancet Neurology 14 (11): 1121-34.

Egaas, B., E. Courchesne, and O. Saitoh. 1995. "Reduced Size of Corpus Callosum in Autism." Archives of Neurology. https://doi.org/10.1001/archneur.1995.00540320070014.

Fatemi, S. Hossein, Kimberly A. Aldinger, Paul Ashwood, Margaret L. Bauman, Charles D. Blaha, Gene J. Blatt, Abha Chauhan, et al. 2012. "Consensus Paper: Pathological Role of the Cerebellum in Autism." Cerebellum 11 (3): 777-807.

Fischl, Bruce, David H. Salat, Evelina Busa, Marilyn Albert, Megan Dieterich, Christian Haselgrove, Andre van der Kouwe, et al. 2002. "Whole Brain Segmentation: Automated Labeling of Neuroanatomical Structures in the Human Brain." Neuron 33 (3): 341-55.

Fischl, B., M. I. Sereno, and A. M. Dale. 1999. “Cortical Surface-Based Analysis. II: Inflation, Flattening, and a Surface-Based Coordinate System.” NeuroImage 9 (2): 195-207.

Grove, Jakob, Stephan Ripke, Thomas D. Als, Manuel Mattheisen, Raymond K. Walters, Hyejung Won, Jonatan Pallesen, et al. 2019. "Identification of Common Genetic Risk Variants for Autism Spectrum Disorder." Nature Genetics 51 (3): 431-44.

Haar, Shlomi, Sigal Berman, Marlene Behrmann, and Ilan Dinstein. 2016. "Anatomical Abnormalities in Autism?" Cerebral Cortex 26 (4): 1440-52.

Hazlett, Heather Cody, Hongbin Gu, Robert C. McKinstry, Dennis W. W. Shaw, Kelly N. Botteron, Stephen R. Dager, Martin Styner, et al. 2012. "Brain Volume Findings in 6-Month-Old Infants at High Familial Risk for Autism." The American Journal of Psychiatry 169 (6): 601-8.

Hodge, Steven M., Nikos Makris, David N. Kennedy, Verne S. Caviness, James Howard, Lauren McGrath, Shelly Steele, Jean A. Frazier, Helen Tager-Flusberg, and Gordon J. Harris. 2010. "Cerebellum, Language, and Cognition in Autism and Specific Language Impairment.” Journal of Autism and Developmental Disorders. https://doi.org/10.1007/s10803-009-0872-7.

Holiga, Štefan, Joerg F. Hipp, Christopher H. Chatham, Pilar Garces, Will Spooren, Xavier Liogier D’Ardhuy, 
Alessandro Bertolino, et al. 2019. "Patients with Autism Spectrum Disorders Display Reproducible Functional Connectivity Alterations." Science Translational Medicine 11 (481).

https://doi.org/10.1126/scitranslmed.aat9223.

Hosmer, David W., and Stanley Lemeshow. 2000. Applied Logistic Regression.

Insel, Thomas R. 2014. "The NIMH Research Domain Criteria (RDoC) Project: Precision Medicine for Psychiatry." The American Journal of Psychiatry 171 (4): 395-97.

Ioannidis, John P. A. 2005. "Why Most Published Research Findings Are False.” PLoS Medicine 2 (8): e124.

Just, M. A. 2004. "Cortical Activation and Synchronization during Sentence Comprehension in High-Functioning Autism: Evidence of Underconnectivity." Brain. https://doi.org/10.1093/brain/awh199.

Klin, A., J. Lang, D. V. Cicchetti, and F. R. Volkmar. 2000. "Brief Report: Interrater Reliability of Clinical Diagnosis and DSM-IV Criteria for Autistic Disorder: Results of the DSM-IV Autism Field Trial." Journal of Autism and Developmental Disorders 30 (2): 163-67.

Kriegeskorte, Nikolaus, W. Kyle Simmons, Patrick S. F. Bellgowan, and Chris I. Baker. 2009. "Circular Analysis in Systems Neuroscience: The Dangers of Double Dipping.” Nature Neuroscience 12 (5): 535-40.

Krumm, Niklas, Tychele N. Turner, Carl Baker, Laura Vives, Kiana Mohajeri, Kali Witherspoon, Archana Raja, et al. 2015. "Excess of Rare, Inherited Truncating Mutations in Autism." Nature Genetics 47 (6): 582-88.

Lange, Nicholas, Brittany G. Travers, Erin D. Bigler, Molly B. D. Prigge, Alyson L. Froehlich, Jared A. Nielsen, Annahir N. Cariello, et al. 2015. "Longitudinal Volumetric Brain Changes in Autism Spectrum Disorder Ages 6-35 Years." Autism Research: Official Journal of the International Society for Autism Research 8 (1): 82-93.

Lee, Sang Hong, Michael E. Goddard, Naomi R. Wray, and Peter M. Visscher. 2012. "A Better Coefficient of Determination for Genetic Profile Analysis." Genetic Epidemiology 36 (3): 214-24.

Lefebvre, Aline, Anita Beggiato, Thomas Bourgeron, and Roberto Toro. 2015. "Neuroanatomical Diversity of Corpus Callosum and Brain Volume in Autism: Meta-Analysis, Analysis of the Autism Brain Imaging Data Exchange Project, and Simulation." Biological Psychiatry 78 (2): 126-34.

Mohammad-Rezazadeh, Iman, Joel Frohlich, Sandra K. Loo, and Shafali S. Jeste. 2016. "Brain Connectivity in Autism Spectrum Disorder." Current Opinion in Neurology. https://doi.org/10.1097/wco.0000000000000301.

Murphy, Declan, and Will Spooren. 2012. "EU-AIMS: A Boost to Autism Research.” Nature Reviews. Drug Discovery 11 (11): 815-16.

Picci, Giorgia, Stephen J. Gotts, and K. Suzanne Scherf. 2016. "A Theoretical Rut: Revisiting and Critically Evaluating the Generalized Under/over-Connectivity Hypothesis of Autism.” Developmental Science 19 (4): 524-49.

Piven, J., S. Arndt, J. Bailey, S. Havercamp, N. C. Andreasen, and P. Palmer. 1995. “An MRI Study of Brain Size in Autism." The American Journal of Psychiatry 152 (8): 1145-49.

Poldrack, Russell A., Chris I. Baker, Joke Durnez, Krzysztof J. Gorgolewski, Paul M. Matthews, Marcus R. Munafò, Thomas E. Nichols, Jean-Baptiste Poline, Edward Vul, and Tal Yarkoni. 2017. "Scanning the Horizon: Towards Transparent and Reproducible Neuroimaging Research.” Nature Reviews. Neuroscience 18 (2): 115-26.

Poldrack, Russell A., Grace Huckins, and Gael Varoquaux. 2019. "Establishment of Best Practices for Evidence for Prediction: A Review.” JAMA Psychiatry, November. https://doi.org/10.1001/jamapsychiatry.2019.3671.

Power, Jonathan D., Kelly A. Barnes, Abraham Z. Snyder, Bradley L. Schlaggar, and Steven E. Petersen. 2012. "Spurious but Systematic Correlations in Functional Connectivity MRI Networks Arise from Subject Motion." NeuroImage 59 (3): 2142-54.

Power, Jonathan D., Alexander L. Cohen, Steven M. Nelson, Gagan S. Wig, Kelly Anne Barnes, Jessica A. Church, Alecia C. Vogel, et al. 2011. "Functional Network Organization of the Human Brain." Neuron 72 (4): 665-78.

Roelofs, Rebecca, Sara Fridovich-Keil, John Miller, Vaishaal Shankar, Moritz Hardt, Benjamin Recht, and Ludwig Schmidt. 2019. "A Meta-Analysis of Overfitting in Machine Learning." In Proceedings of the 33rd International Conference on Neural Information Processing Systems, 9179-89. papers.neurips.cc.

Sandin, Sven, Paul Lichtenstein, Ralf Kuja-Halkola, Christina Hultman, Henrik Larsson, and Abraham Reichenberg. 2017. "The Heritability of Autism Spectrum Disorder." JAMA: The Journal of the American Medical Association 318 (12): 1182-84.

Schizophrenia Working Group of the Psychiatric Genomics Consortium. 2014. "Biological Insights from 108 Schizophrenia-Associated Genetic Loci." Nature 511 (7510): 421-27.

Steyerberg, Ewout W., and Frank E. Harrell Jr. 2016. "Prediction Models Need Appropriate Internal, Internal-External, and External Validation." Journal of Clinical Epidemiology 69 (January): 245-47.

Traut, Nicolas, Anita Beggiato, Thomas Bourgeron, Richard Delorme, Laure Rondi-Reig, Anne-Lise Paradis, and 
Roberto Toro. 2018. "Cerebellar Volume in Autism: Literature Meta-Analysis and Analysis of the Autism Brain Imaging Data Exchange Cohort." Biological Psychiatry 83 (7): 579-88.

Varoquaux, Gaël. 2018. "Cross-Validation Failure: Small Sample Sizes Lead to Large Error Bars." NeuroImage 180 (Pt A): 68-77.

Varoquaux, Gaël, Flore Baronnet, Andreas Kleinschmidt, Pierre Fillard, and Bertrand Thirion. 2010. "Detection of Brain Functional-Connectivity Difference in Post-Stroke Patients Using Group-Level Covariance Modeling." Medical Image Computing and Computer-Assisted Intervention: MICCAI ... International Conference on Medical Image Computing and Computer-Assisted Intervention 13 (Pt 1): 200-208.

Varoquaux, Gael, Alexandre Gramfort, Fabian Pedregosa, Vincent Michel, and Bertrand Thirion. 2011. "Multi-Subject Dictionary Learning to Segment an Atlas of Brain Spontaneous Activity." Information Processing in Medical Imaging: Proceedings of the ... Conference 22: 562-73.

Weiner, Daniel J., Emilie M. Wigdor, Stephan Ripke, Raymond K. Walters, Jack A. Kosmicki, Jakob Grove, Kaitlin E. Samocha, et al. 2017. "Polygenic Transmission Disequilibrium Confirms That Common and Rare Variation Act Additively to Create Risk for Autism Spectrum Disorders." Nature Genetics 49 (7): 978-85.

Wolff, Jason J., Guido Gerig, John D. Lewis, Takahiro Soda, Martin A. Styner, Clement Vachet, Kelly N. Botteron, et al. 2015. "Altered Corpus Callosum Morphology Associated with Autism over the First 2 Years of Life." Brain: A Journal of Neurology 138 (Pt 7): 2046-58.

Wolpert, David H. 1992. "Stacked Generalization.” Neural Networks. https://doi.org/10.1016/s0893-6080(05)80023-1.

Woo, Choong-Wan, Luke J. Chang, Martin A. Lindquist, and Tor D. Wager. 2017. "Building Better Biomarkers: Brain Models in Translational Neuroimaging." Nature Neuroscience 20 (3): 365-77.

Woolrich, Mark W., Saad Jbabdi, Brian Patenaude, Michael Chappell, Salima Makni, Timothy Behrens, Christian Beckmann, Mark Jenkinson, and Stephen M. Smith. 2009. "Bayesian Analysis of Neuroimaging Data in FSL." NeuroImage 45 (1 Suppl): S173-86.

Wray, Naomi R., Jian Yang, Michael E. Goddard, and Peter M. Visscher. 2010. "The Genetic Interpretation of Area under the ROC Curve in Genomic Profiling." PLoS Genetics 6 (2): e1000864.

Zielinski, Brandon A., Molly B. D. Prigge, Jared A. Nielsen, Alyson L. Froehlich, Tracy J. Abildskov, Jeffrey S. Anderson, P. Thomas Fletcher, et al. 2014. "Longitudinal Changes in Cortical Thickness in Autism and Typical Development.” Brain: A Journal of Neurology 137 (Pt 6): 1799-1812. 